\title{
A SOA-based Framework for Integration of Intelligent Rock Bolts with Internet of Things
}

\author{
Jens Eliasson, Jerker Delsing, Asma Raayatinezhad, and Rumen Kyusakov \\ Department of Computer Science, Electrical and Space Engineering, \\ Luleå University of Technology, Luleå, Sweden. \\ Email: jens.eliasson@1tu.se
}

\begin{abstract}
The mining industry is seeing a bright future with a high demand for minerals and relatively high material prices. Much of this is driven by the rapid industrial expansion in countries such as China and others. However, even though the mining business seems promising, there are some important issues that must be addressed in order to maintain a costeffective and competitive edge. Worker and environment safety, working conditions, are very important issues. Another issue is attracting skilled personal to work in the deep mines of the future. Today, the mining industry has problems hiring personnel, due to hazardous working environments and re-locating to the distant regions. If the workers' safety could be increased, attracting skilled personnel would become one less problem. Rock bolts and shotcrete are in use today to reinforce tunnel walls, and expensive measurement devices are used in order to monitor seismic activity and rock stress. However, by not having realtime monitoring of rock bolts makes it difficult to monitor them. A better approach would be to make rock bolts intelligent and have them measure stress and seismic activity. Furthermore, by connecting the rock bolts to a network, real-time monitoring of them is made feasible.

In this paper, we propose the merger of the Internet of Things (IoT) approach with traditional rock bolts, thereby allowing online monitoring of rock bolt status. This enables an increased possibility to enhance work safety by being able to detect anomalies on the rock earlier, thereby giving workers and machinery an earlier alarm to evacuate hazardous locations in the mine.
\end{abstract}

Index Terms-Internet of Things, SOA, rock bolts, sensor networks

\section{INTRODUCTION}

The mining industry is seeing a bright future with a high demand for minerals and relatively high material prices. Much of this is driven by the rapid industrial expansion in countries such as BRIC (Brazil, Russia, India, and China) as well as others [1]. A report from Deloitte [2] stated that some of the most crucial issues for the mining industry are:

- Labour pains indicating that there is a severe problem in the mining industry to attract competent personnel. Hazardous working environments is a real obstacle in the work of recruiting today's younger work force.

- Legislative Olympics meaning that laws are regulations are becoming tougher to comply with in the mining business. Environmental and social issues are influencing more and more on the mining companies and their business.

- Restless stakeholders demand heightened corporate social responsibility meaning that corporations need to take a larger social responsibility for the communities that they operate in.

In traditional deep mining, tunnels and caverns are created by first drilling small but deep holes. Then the holes are filled with explosives which in turn are detonated in a specific order to achieve a maximum blasting effect. After the explosion, haulers are used to carry the big pieces of rock containing ore for further processing. When the space is emptied, machines are used to spray shotcrete [3], a kind of liquid cement. The shotcrete helps stabilizing the outer walls of a tunnel, and can be used either as-is or with fiber-reinforcements. The fibers are usually small strips of metal which helps preventing cracks in the shotcrete. Metal wire nets can also be used to reinforce the shotcrete even more.

Rock bolts play on important role on securing tunnels and open spaces. Rock bolts have been in use since the 1890's or so. Rock bolts transfer the load on more unstable tunnel wall to the more rigid rock deeper in the rock, as investigated by Malmgren in [4]. Rock bolts are exposed to different types of forces such as push-pull and skewing, and may be of different design, i.e. mechanical or even fiber glass. Even though rock bolts have been in use more than a hundred years, and now comes in different flavors, they all lack in one aspect. The only mean of detecting the forces that are on a bolt is by visual inspection. A recent work by Chengyi et al. [5] presents a design of microcontroller-based solution for rock bolt monitoring that uses infrared communication. Even though devices such as geophones and seismic devices are now in use by the mine industry, rock bolts are still based on a very old concept.

To further stabilize the outer wall, rock bolts are used to transfer forces from the outer wall to the raw rock a few meters inside the rock. A rock bolt is usually about an inch wide and several meters long. Rock bolts are installed after the shotcrete, and can also be used to fixate a metal net to prevent rocks to fall down on workers or equipment. When an earthquake occurs, shock waves travel inside the rock surrounding the tunnels of a mine. When the waves hit open spaces, e.g. tunnels etc., they reverse direction and can cause massive damage on shotcrete and rock bolts. This can be seen as cracks of the shotcrete, collapsed sections of shotcrete or damaged rock bolts. However, only the rock bolt's head can be inspected visually. If the part of a rock bolt that is deep inside the rock is damaged there are no means of inspecting it. 
A better approach would be to have constant monitoring of all rock bolts in use in a mine. However, this would require power and data cables to be used to interconnect thousands of rock bolts in a very unstable environment. A more feasible solution is to use wireless communication since this eliminates the need of costly and time consuming cable installations. In [6], Jiang et al.showed a wireless sensor network equipped with cameras for mine monitoring.

The use of SOA in industrial applications has been well investigated. In [7], Colombo et al. presents current state of the art regarding the use of distributed services where top-level systems such as business management can be interconnected with systems used on the factory floor.

In this paper, we propose an integration of Internet of Things (IoT) [8] with traditional rock bolts, thereby allowing online monitoring of rock bolt status. This enables an increased possibility to enhance work safety by being able to detect anomalies on the rock earlier, thereby giving workers and machinery an earlier alarm to evacuate hazardous locations in the mine. By utilizing the service-oriented approach, service and device discovery comes automatically as well as zeroconfiguration networking where devices, such as intelligent rock bolts, can automatically download settings and thereby configure themselves. This precludes the need of a timeconsuming manual installation, and minimized the possibilities of human errors. The use of SOA in other industrial applications such as process monitoring and control as been investigated by Karnouskos et al. [9] and Jammes et al. [10].

This paper is outlined as follows: Sections II and III present enabling technologies and the the proposed architecture. Results from this work is presented in Sec. IV. After that future work is outlined in Section V, followed by conclusions and acknowledgement in Sections VI and VII, respectively.

\section{ENABLING TECHNOLOGIES}

In this section, a number of enabling technologies are presented together with their characteristics.

\section{A. Rock bolts}

Different types of roof support elements are being used in mining industry. One of the most important of them is the rock bolt. It is a long metal bar with three specific anchor types. It is employed to stabilize the roof and prevent it from collapsing. Among the different methods of rock mass stabilization, the use of rock bolt is the most efficient way. The role of rock bolt is to connect stones that have normal joints and gaps, or connecting discontinuous rock mass together like the sedimentary rocks which are layered plates. Generally, rock bolt is exerted in excavation in tunnel building and mining industry to fixate rock, stone, and other dense natural materials. A sample rock bolt application in mine industry is shown in Fig. 1.

\section{B. Wireless communication platform}

A wireless sensor network is composed of a large number of heterogeneous sensor nodes, or sources, that sense phenomena

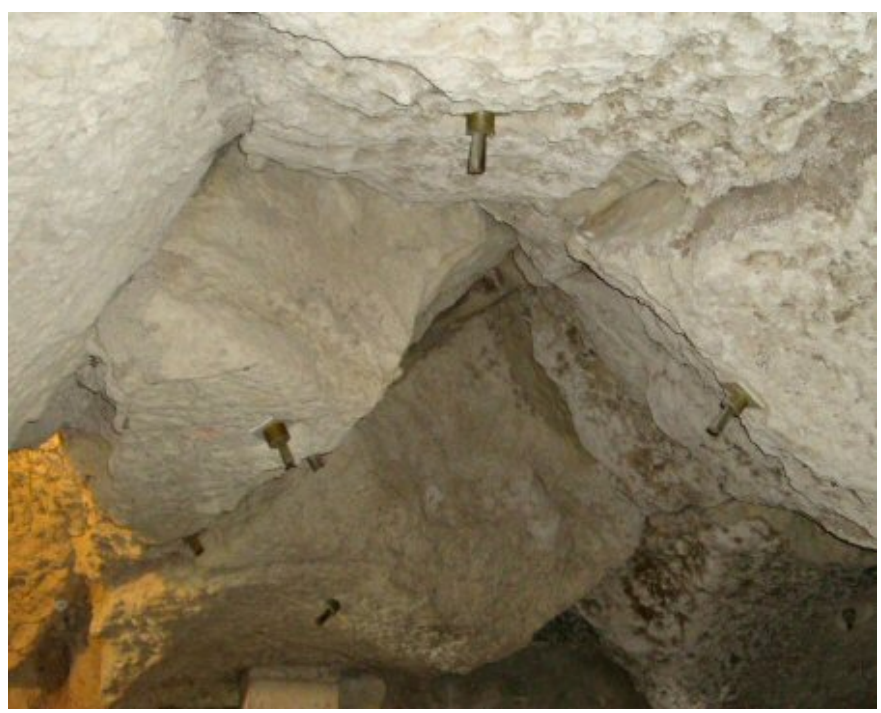

Fig. 1. Rock bolts in mine.

in the physical world. Sensor networks also include gateways, or sinks, whose task is to forward sensor data from nodes in the internal network to an external network [11]. Research on WSN technology originally targeted military applications, such as battlefield surveillance, land mine detection, and soldier monitoring. Current research on wireless sensor networks is also motivated by an increasing number of civil usage scenarios, such as environmental issue, home automation, energy efficiency and others.

Internet of Things is a term used in computer science. The term refers the concept of small Internet-connected sensors and actuators [12] embedded in the physical world. The IoT approach enables monitoring and control of physical phenomena such as temperature, vibration, humidity etc. [13].

In order to use low power in wireless sensors, 6LoWPAN is used in this research. 6LoWPAN is defined as IPv6 over IEEE 802.15.4 (ZigBee) [14]. It is the standard for low data rate, low power, and low cost wireless personal area network [15].

To provide routing between wireless sensors and efficient low power communication links, RPL protocol is used. RPL is an IPv6-based routing protocol for low power and lossy networks [16]. Low power and Lossy networks (LLNs) are made up of many embedded devices which are limited in resources such as battery, memory and processing [17].

\section{Service-oriented architecture}

Service-oriented architecture (SOA) is an approach for developing and managing complex distributed systems where the functionality of the systems is strictly separated into selfcontained functional blocks called services. By using loosely coupled components, described by Interface Description Languages (IDL), that can be discovered dynamically and communicate in an event-based form, the system becomes amendable and allows for higher flexibility. These properties will be of 


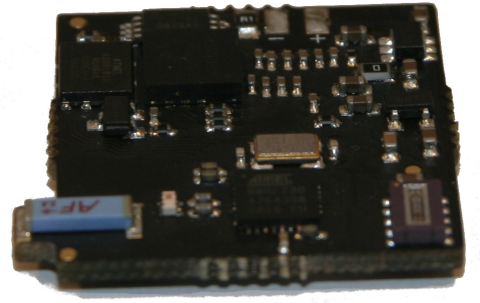

Fig. 2. Mulle sensor platform

high importance for the future mining industry that will rely more and more on large number of heterogeneous devices used thorough out the infrastructure of the mines: tunnels, mobile autonomous vehicles, robots etc.

SOA is seen as a promising technique to bridge the gap between various industrial devices and enterprise applications [18]. The closer integration between ubiquitous embedded systems such as wireless sensor nodes and high-end systems could lead to higher flexibility in process optimization and evolution [19]. A key element in this architecture is the use of common communication protocols on all levels of the process chain. An established technology for implementing SOA is the web service stack that is based on TCP/IP, DNS, HTTP, XML and other web technologies. As the deployment of the web services on resource-constrained devices leads to unacceptable overhead in terms of RAM, CPU and network usage the use of intermediate components such as gateways or mediators is often required. However, the introduction of additional hardware and software modules to the system increases its complexity and maintenance costs. As a consequence, a number of standardization activities within $\mathrm{W} 3 \mathrm{C}$ and IETF are developing new web service technologies that can provide the required efficiency for resource constrained devices [20]. These emerging technologies are in the core of our architecture and include: 6LoWPAN, Constrained Application Protocol CoAP) and the related technologies from the IETF CoRE working group as well as Efficient XML Interchange (EXI) format for representing structured information. Kyusakov et al. showed in [21] how SOA can be applied even to small resource-constrained devices.

\section{CoAP (Constrained Application Protocol)}

The IETF Constrained Application Protocol is an application-layer protocol designed to provide web services working with constrained nodes - devices using microcontrollers with small amounts of ROM and RAM, running 6LoWPAN network stacks with high packet error rates etc. The protocol is designed for low-power networking allowing the nodes to switch to sleep mode to extend their battery life. CoAP provides a request/response interaction model between application end-points, supports built-in discovery of services and resources, and includes key concepts of the Web such as URIs, RESTful interaction, extensible header options, etc. CoAP easily interfaces with HTTP for integration with the Web while meeting specialized requirements such as multicast support, very low overhead and simplicity for constrained environments. CoAP runs over UDP unlike HTTP which is based on TCP.

\section{INTELLIGENT ROCK BOLT ARCHITECTURE}

This section presents the design of the intelligent rock bolt and gives a detailed overview of the service and communication architecture. The architecture consists of a rock bolt, sensors and a Mulle wireless sensor node [22]. The backendsystem is composed of several services and servers.

\section{A. Sensor node}

The Intelligent rock bolt consists of several components - a sensor board and a Mulle v6.2 wireless sensor node. See Fig. 3 for a schematic view of the architecture. The Mulle runs the Contiki operating system and communicates using 6LoWPAN and CoAP.

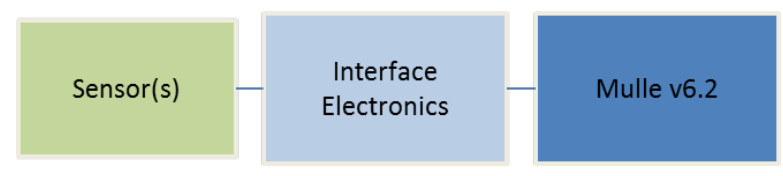

Fig. 3. Intelligent rock bolt system

The Mulle interfaces the rock bolt [23], shown in Fig. 4, through an interface boards which contains all sensors and interfaces required for monitoring the bolt. Communication is performed using 6LoWPAN on the $868 \mathrm{MHz}$ band, by the use of the CoAP protocol. All sensors are analog, and high performance amplifiers are used to amplify the very weak signals that are produced by the sensors. High-resolution analog-to-digital converters are used to sample the signals. The interface board also contains power management features. The Mulle features an on-board 2MB memory for data storage which can be used to buffer measurements prior to data transmission.

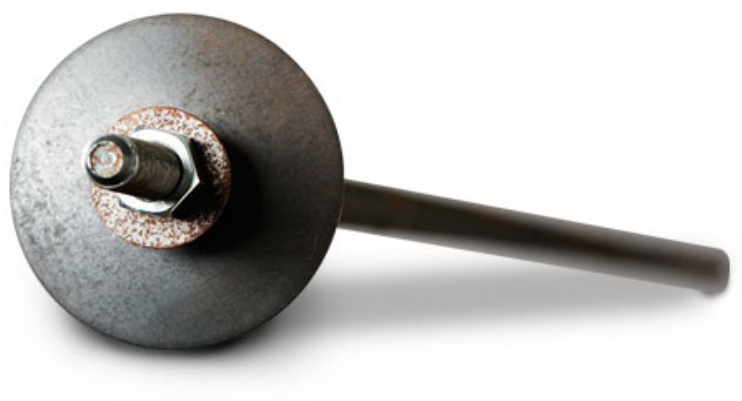

Fig. 4. Rock bolt used in research 


\section{B. Service-oriented architecture}

Integration between back-end services, legacy systems and rock bolt-based sensors is based on the SOA approach. Each sensor is connected to a wireless network, potentially using mesh (multi-hop) configuration, using 6LoWPAN, and can communicate with cloud-based services inside the company network or on the public cloud (Internet). The server is composed of several services which can be integrated with sensor nodes, mobile devices, or can be distributed on cloud infrastructure.

In Fig. 5, the SOA architecture is shown. Services are deployed on sensor nodes (rock bolts), on gateways or in the cloud. Some services must of course be deployed on sensors in order to access the physical sensors. However, filtering services can for example be deployed at any level of the architecture.

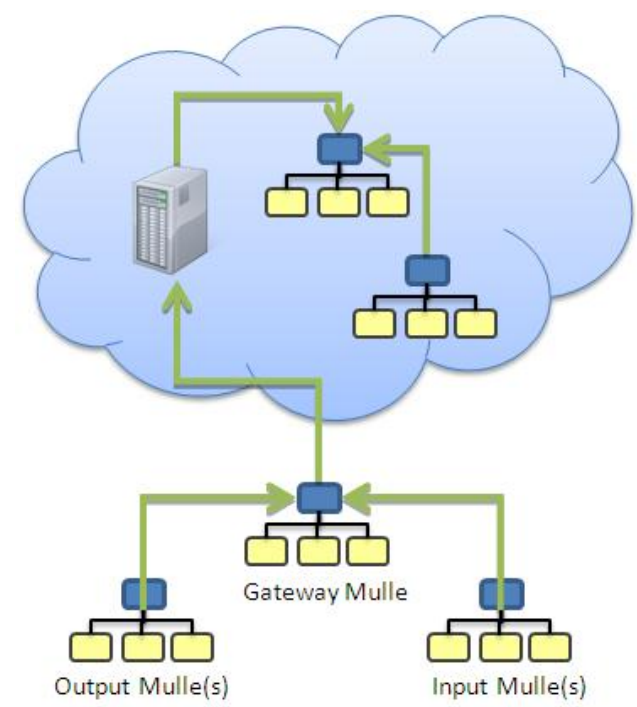

Fig. 5. Communication architecture

Some services that have been implemented for the proposed platform are listed below together with their functionality:

1) Configuration - is used by all intelligent rock bolts in order to get all available configuration parameters.

2) Time - is used to synchronize sensor platforms using CoAP, comparable with the NTP protocol.

3) Data acquisition - access to sensor data.

4) Proxy - facilitates the communication with the sensors and other mobile devices.

5) Filter - analyzes and filters sensor data, either on device or in the cloud.

6) Alarm - based on certain rules it warns the user for possible critical conditions through a SMS, email or other mechanisms.

7) Historian - collects sensor data and stores it in an SQL database for further processing and/or visualization.

The server has the ability to communicate with the devices in different types of formats such as text, binary, XML, and

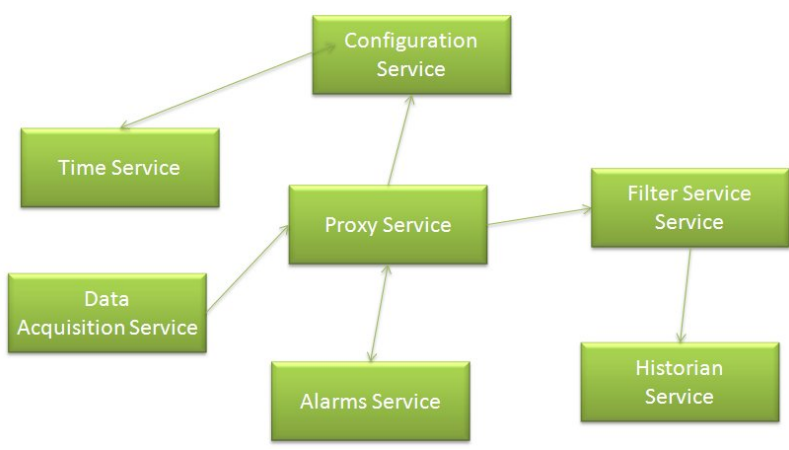

Fig. 6. Services' deployment

EXI. The advantage of the text format is its convenience when reading, but it is hard to parse. Binary is efficient to communicate, but requires external tools to make it readable by users. XML is understandable and very well structured, but the size of its messages is big and it is much worse to parse compared to binary formats. EXI has smaller size and it is very efficient to process and easy to transfer. The server supports both IPv6 and IPv4.

The server is based on the open source server implementation part of libcoap [24]. It has been extended with a pluginbased architecture where services can be dynamically loaded and unloaded. All services are loaded using dynamic linking mechanism. An XML-based configuration file controls which services should be loaded during startup. Storage of sensor data and configuration is managed by an MySQL database server. The main interdependencies among the services are shown in Fig. 6.

\section{RESUlTS}

The architectural components shown below have been successfully implemented in the proposed approach. Even though more research is needed, these achievements indicate that the proposed approach is feasible and realistic.

\section{A. Measurement system}

An electronic measurement system, displayed in Fig 7, has been developed on order to be able to monitor a rock bolt. The measurement system is capable of measuring stress/strain as well as vibrations. A strain gauge is used to measure forces to the rock bolt, and a high-performance accelerometer is used to measure vibrations. The measurement system is connected to a Mulle v6.2 sensor platform using the available 60-pin high density expansion port. The measurement system consists of a high-performance AD-converter, amplifiers, and the mentioned sensors.

\section{B. Rock bolt integration}

A first integration test has been performed using a standard rock bolt, the measurement system and a Mulle platform. The Mulle platform, with the measurement system were mounted on the bolt's head. This placement is necessary for the wireless communication to work. Later on, when tests are 


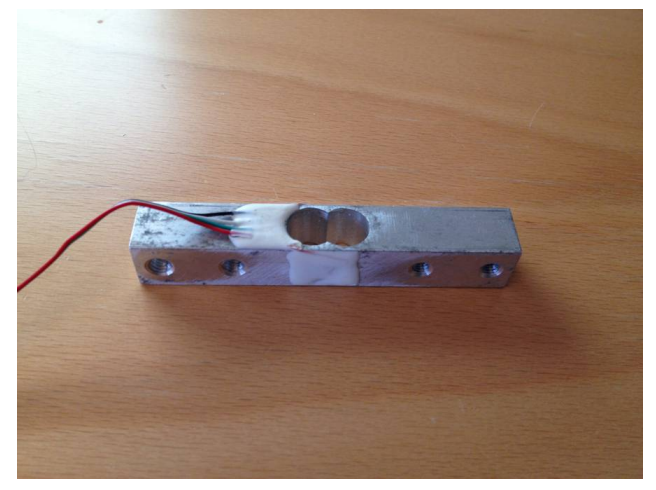

Fig. 7. Strain gauge sensor with bolt integration mount

to be performed in a mine environment, placing an antenna deep inside a mine tunnel wall will drastically block out any wireless transmission.

Tests were performed in order to validate that applied forces to the rock bolt is detected by the Mulle. It has been verified that a rock bolt can be equipped with sensors for status monitoring. However, real field-trials are required in order to fully very the design. In this early stage of the work focus has been on developing the complete architecture. In the next step, real measurement data will be collected at a mine installation.

\section{Wireless sensor platform}

The sensor node used during this work is Eistec's Mulle v6.2 platform equipped with an $868 \mathrm{MHz}$ IEEE 802.15.4 radio transceiver. The Mulle was running the Contiki operating system during the integration tests, but TinyOS with 6LoWPAN is supported as well. All measurements were sampled by the Mulle and written on its serial port for further analysis by a computer. Later on, all signal processing will be performed by the host micro-controller on the Mulle and only processed information will be transmitted using the wireless network. This will reduce the power consumption to a minimum since it is much more efficient to process data than to transmit it, especially in a multi-hop network.

\section{SOA architecture}

A CoAP-based service architecture has been successfully implemented using libcoap [25] on Linux platform. All services run on a modified version of the default server software in libcoap. The server has been modified to allow dynamic loading of services, i.e. plugin-based. All services can be used in event-based mode where no polling is used. This mitigates communication overhead since the unnecessary information need to be transmitted. The implemented services enable a Mulle to obtain a configuration, synchronize its clock, and store sensor data in a MySQL database. The SOA architecture will also be used later on to integrate the intelligent rock bolts with suitable traditional mine stress monitoring systems.

\section{FUTURE WORK}

The research presented in this paper is the first step towards an ultra-low power Internet-connected intelligent rock bolt.
More work is needed in order to realize this vision, where a dense sensor network consisting of a large number of intelligent rock bolts can be used to monitor the mine in order to get an early warning on pending rock collapse.

Based on the results already obtained, and lessons learned, the research will continue on addressing research questions such as: measurement issues, networking problems including quality of service (QoS) and real-time data transmission, and signal processing.

In the next steps, we will focus on low-power signal processing methods for reducing the data amounts that must be sent from the bolts to a back-end system for analyses. Another issue to investigate is standards for rock bolt (wireless) communication. We will also focus on developing suitable methods for service composition and orchestration so that services available on rock bolts, in the network or in the cloud can be combined in order to create new services and on realtime communication for time-sensitive services.

Tests are planned to be performed in a real mine where important measurement data will be collected. The data will be processed in order to find suitable signal processing algorithms that can be used on a rock bolt. Signal processing will be a key feature for robust real-time monitoring [26]. The newest generation of the Mulle platforms, with better signal processing capabilities, will enable more advanced signal processing algorithms to be used.

\section{Conclusions}

In this paper, the first steps toward an online rock bolt for the intelligent deep mine of the future have been taken. The intelligent rock bolt, consisting of a traditional rock bolt equipped with sensors, actuators, signal processing and wireless communication, can be used to monitor the mine's stability in near real-time. We envision that dynamic stresses in the rock can be measured and analyzed. By using these results, systems for early warnings about pending collapses can be developed in order to broadcast alarms to the management and even workers in the mine. This new architecture can greatly improve working conditions and personnel safety as well as increase mine productivity since more information is available to the management about mine conditions and status.

\section{ACKNOWLEDGEMENT}

The authors would like to thank for their support the European Commission, and the partners of the two EU FP7 projects IMC-AESOP (www.imc-aesop.eu) and I2Mine (http://www.i2mine.eu) for fruitful discussions.

\section{REFERENCES}

[1] P. Kim and A. Tomohiro, "Oil and metal price movements and bric macroeconomy: an empirical analysis," International Journal of Business and Globalisation, vol. 8, pp. 187 - 206, jan 2012.

[2] Deloitte, Tracking the trends 2012. Deloitte, 2012, http://www.miningjournal.com/reports/read-more-deloitte-tracking-the-trends-2012.

[3] E. Hoek, P. K. Kaiser, and W. F. Bawden, Support of underground excavations in hard rock. Mining Research Directorate and Universities Research Incentive Fund, 1993.

[4] L. Malmgren, "Interaction between shotcrete and rock - experimental and numerical study," Ph.D. dissertation, LTU, 2005. 
[5] W. Chengyi, G. Xiumei, and C. Jianming, "Design of novel rock bolt dynamometer based on microcontroller and low-power technology," in Computer Science-Technology and Applications, 2009. IFCSTA '09. International Forum on, vol. 3, dec. 2009, pp. 199 -202.

[6] H. Jiang, L. Chen, J. Wu, S. Chen, and H. Leung, "A reliable and high-bandwidth multihop wireless sensor network for mine tunnel monitoring," Sensors Journal, IEEE, vol. 9, no. 11, pp. 1511 -1517, nov. 2009.

[7] A. Colombo, F. Jammes, H. Smit, R. Harrison, J. Lastra, and I. Delamer, "Service-oriented architectures for collaborative automation," in Industrial Electronics Society, 2005. IECON 2005. 31st Annual Conference of IEEE, nov. 2005, p. 6 pp.

[8] A. Dunkels, J. Eriksson, N. Finne, F. Osterlind, N. Tsiftes, J. Abeille, and M. Durvy, "Low-power ipv6 for the internet of things," in Networked Sensing Systems (INSS), 2012 Ninth International Conference on, june 2012, pp. $1-6$.

[9] S. Karnouskos, A. Colombo, F. Jammes, J. Delsing, and T. Bangemann, "Towards an architecture for service-oriented process monitoring and control," in IECON 2010 - 36th Annual Conference on IEEE Industrial Electronics Society, nov. 2010, pp. $1385-1391$.

[10] F. Jammes, B. Bony, P. Nappey, A. W. Colombo, J. Delsing, J. Eliasson, R. Kyusakov, S. Karnouskos, P. Stluka, and M. TIlly, "Technologies for soa-based distributed large scale process monitoring and control systems," in Proceeding IEEE IECON 2012: 38th Annual Conference of the IEEE Industrial Electronics Society, Oct. 2012, pp. 5803-5808.

[11] D. Estrin, "Wireless sensor networks: application driver for low power distributed systems," in Low Power Electronics and Design, International Symposium on, 2001., 2001, p. 194.

[12] O. Vermesan and P. Friess, Internet of Things - Global Technological and Societal Trends from Smart Environments and Spaces to Green Ict, ser. River Publishers series in communications. River Publishers, 2011, http://books.google.se/books?id=dhCHZwEACAAJ.

[13] G. Kortuem, F. Kawsar, D. Fitton, and V. Sundramoorthy, "Smart objects as building blocks for the internet of things," Internet Computing, IEEE, vol. 14 , no. 1, pp. 44-51, 2010.

[14] Z. Shelby and C. Bormann, 6LoWPAN: The Wireless Embedded Internet, ser. Wiley Series on Communications Networking \& Distributed Systems. John Wiley \& Sons, 2010, http://books.google.se/books?id=w90yHNrpBqsC.
[15] G. Mulligan, "The 6lowpan architecture," in Proceedings of the 4th workshop on Embedded networked sensors. ACM, 2007, pp. 78-82.

[16] L. Saad and B. Tourancheau, "Sinks mobility strategy in ipv6-based wsns for network lifetime improvement," in New Technologies, Mobility and Security (NTMS), 2011 4th IFIP International Conference on, feb. 2011, pp. $1-5$

[17] H. Kermajani and C. Gomez, "Route change latency in low-power and lossy wireless networks using rpl and 6lowpan neighbor discovery," in Computers and Communications (ISCC), 2011 IEEE Symposium on, 28 2011-july 12011 , pp. $937-942$.

[18] S. Karnouskos, D. Guinard, D. Savio, PatrikSpiess, O. Baecker, V. Trifa, and L. M. S. deSouza, "Towards the real-time enterprise: Servicebased integration of heterogeneoussoa-ready industrial devices with enterprise applications," in Proceedings of the 13th IFAC Symposium on Information Control Problemsin Manufacturing (INCOM)., Moscow, Russia, Jun. 2009.

[19] S. Karnouskos, A. W. Colombo, T. Bangemann, K. Manninen, R. Camp, M. TIlly, F. Jammes, J. Delsing, and J. Eliasson, "A soa-based architecture for empowering future collaborative cloud-based industrial automation," in Proceedings IEEE IECON 2012, Oct. 2012, pp. 57705775.

[20] Z. Shelby, "Embedded web services," Wireless Communications, IEEE, vol. 17, no. 6, pp. $52-57$, december 2010.

[21] R. Kyusakov, J. Eliasson, J. Delsing, J. van Deventer, and J. Gustafsson, "Integration of wireless sensor and actuator nodes with IT infrastructure using service-oriented architecture," Industrial Informatics, IEEE Transactions on, vol. PP, no. 99, p. 1, 2012.

[22] J. Johansson, M. Völker, J. Eliasson, Å. Östmark, P. Lindgren, and J. Delsing, "Mulle: A minimal sensor networking device - implementation and manufacturing challenges," in Proceedings IMAPS Nordic, 2004, pp. 265-271.

[23] "Nybergs mekaniska verkstad ab - rock bolt nmx," 2012.

[24] K. Kuladinithi, O. Bergmann, T. Pötsch, M. Becker, and C. Görg, "Implementation of coap and its application in transport logistics," Proc. IP+ SN, Chicago, IL, USA, 2011.

[25] "libcoap," Nov. 2012, http://libcoap.sourceforge.net/

[26] J. Cheng, X. Sun, D. Liu, J. Bian, and D. Zou, "Reducing measurement noise in rock bolts detecting," in Electronic Measurement Instruments, 2009. ICEMI '09. 9th International Conference on, aug. 2009, pp. 4-527 $-4-531$. 